\title{
Patchiness and duodenal-jejunal variation of the mucosal abnormality in coeliac disease and dermatitis herpetiformis
}

\author{
B. B. SCOTT AND M. S. LOSOWSKY \\ From the University Department of Medicine, St. James's Hospital, Leeds
}

SUMMARY The incidence and degree of patchiness of mucosal abnormality in both coeliac disease (CD) and dermatitis herpetiformis (DH) is documented. As judged by both stereomicroscopy and subjective histology, patchiness occurred frequently in both $\mathrm{CD}$ and DH patients. In most cases the difference of abnormality was of only one grade, but in approximately $25 \%$ as assessed by stereomicroscopy and $10 \%$ as assessed by histology the difference was of two or more grades. In control subjects with normal small bowel mucosa the variation of the mucosal appearance between the duodenum and proximal jejunum was studied. Contrary to popular belief, no significant difference of villous and crypt measurements or of apparent villous 'bridging' and 'branching' between these two sites was found, if only well-orientated sections were studied. The stereomicroscopic appearances were also similar at these two sites, although villi tended to be broader in the duodenal biopsies. The duodenal-jejunal variation was also studied in CD and DH patients and, although by both stereomicroscopy and subjective histology the appearances were similar in most patients, in approximately $33 \%$ the duodenal abnormality was the more severe and, surprisingly, the jejunal abnormality was more severe in approximately $15 \%$. It is concluded that multiple, precisely located biopsies of both the duodenum and proximal jejunum are invaluable in the investigation of small bowel disease and in assessing response to treatment.

Coeliac disease (CD) may be defined as ' . . the condition in which there is an abnormal jejunal mucosa that responds morphologically to treatment with a gluten-free diet' (Booth, 1970). Thus criteria for recognition of improvement are crucial.

It is known that the mucosal lesion of $C D$ varies with the level (MacDonald et al., 1964;Stewart et al., 1967) and thus improvement must be assessed at the same level. Obviously to assess improvement at a particular level one must first determine the degree of any variation in morphology - that is, patchiness -which might occur at the same level. It is apparently widely assumed that there is no significant patchiness, as most published reports of CD patients responding morphologically to treatment are based on the evidence of single biopsies. With the advent of suitable instruments for taking multiple biopsies (Brandborg et al., 1959; Baker and Hughes, 1960; Flick et al., 1961), it has been possible to assess

Received for publication 4 August 1976 patchiness, but apparently no formal study of this has been undertaken. There are divergent opinions about patchiness, some leading workers in North America denying its existence in untreated CD (Jeffries et al., 1968; Brow et al., 1971; Gebhard et al., 1974), whereas many isolated cases of this have been reported from the United Kingdom (Holmes et al., 1961; Hourihane, 1963; Scott et al., 1964; Spiro et al., 1964; Roy-Choudhury et al., 1966; Girdwood et al., 1966; Thompson, 1974). In DH there is less disagreement and some have taken the existence of patchiness as evidence for a different mucosal lesion in that condition (Brow et al., 1971; Gebhard et al., 1974).

The above definition of $\mathrm{CD}$ specifies a response of the jejunal mucosa and, indeed, the site of biopsy preferred by most workers and recommended by Rubin and Dobbins (1965) is the jejunum just past the duodenojejunal junction (ligament of Treitz). However, many studies have relied on duodenal biopsies which are obtained more readily especially in children (Rubin et al., 1960) and thus it is import- 
ant to assess the value of duodenal biopsies in the diagnosis of CD. Various differences between biopsies from these two sites have been claimed in control subjects (Roy-Choudhury et al., 1966; Rubin et al., 1960; Broitman et al., 1970) and Rubin (1961) has recommended that the proximal three-quarters of the duodenum be avoided bcause of mild nonspecific abnormalities. However, one study (Stewart et al., 1967) has shown no significant difference in the appearance of biopsies taken from the lower duodenum and upper jejunum. A formal comparison between duodenal and jejunal biopsies in control subjects is required.

As the mucosal abnormality in CD tends to be more severe proximally (MacDonald et al., 1964; Stewart et al., 1967), a mild coeliac lesion might be missed if only the proximal jejunum is sampled. Such a possibility is suggested by the results of the full length study of MacDonald et al. (1967) in 11 patients, although the exact sites of the jejunal biopsies were not given. Stewart et al. (1967), however, found no difference between duodenal and jejunal biopsies in four patients but in only two was the jejunal biopsy taken from the first loop of jejunum. There is, therefore, also a need for a comparison of duodenal and jejunal biopsies in $\mathrm{CD}$ to determine whether additional information can be gained by duodenal biopsy. This is particularly so as there is an increasing awareness of the wide clinical spectrum of $C D$, and patients are being detected with very mild disease, especially in family studies (Robinson et al., 1971; Stevens et al., 1975) and in patients with dermatitis herpetiformis (Weinstein, 1974).

This study was therefore designed to document the incidence and degree of patchiness in CD and $\mathrm{DH}$, to compare duodenal with jejunal biopsies in control subjects, and to assess variation between these two sites in patients with CD and DH. Because of the large number of biopsies, it was not practicable to study them all by objective histological measurements and most were therefore studied just by stereomicroscopy and by blind subjective histological assessment after validation of that technique as described by Rubin et al. (1960).

\section{Methods}

\section{BIOPSY ASSESSMENT}

Biopsies were taken under fluoroscopic control from the distal end of the second part of the duodenum and from just past the duodenojejunal junction, with the hydraulic multiple biopsy instrument (Quinton Instruments, Seattle). They were assessed by stereomicroscopy, subjective histology, and, in some cases, by objective histological measurements as follows:
Stereomicroscopy All biopsies were graded by one of the authors, within minutes of obtaining them, as villous (distinct finger or leaf-shaped structures with the long diameter less than 10 times the shorter), ridged (the long diameter more than 10 times the shorter), convoluted (ridges containing a minimum of two angulations of $90^{\circ}$ or less), or flat (no villi, ridges, or convolutions present).

Subjective histology The stained sections of the biopsies were graded as grade 1 (normal, no shortening of villi or lengthening of crypts); grade 2 (slight partial villous atrophy; slight shortening of villi); grade 3 (marked partial villous atrophy; marked shortening of villi); grade 4 (subtotal villous atrophy, no definite villous structure). The subjective assessments were performed blindly by one of the authors-that is, the slides were randomly mixed and presented without any identification.

Objective assessment The stained sections were projected at a magnification of 500 on to a screen. The best orientated part of the specimen was selected for study, this usually consisting of five or six villi in the middle of the section. Poorly orientated sections were discarded. The following two measurements were made: (1) total mucosal thickness (M)the distance from the muscularis mucosae to the mean position of the villous tips or to the flat surface in the case of subtotal villous atrophy, and (2) the crypt depth (C)-the distance from the muscularis mucosae to the dilatation of the crypt at the start of a villus. The average measurement of all wellexposed crypts was taken (usually three). The following indices were calculated from these measurements: (1) mean villus height (V) - that is, the difference between total mucosal thickness (M) and the mean crypt depth (C), (2) the ratio of villus height to crypt depth $(\mathrm{V} / \mathrm{C})$, and (3) the ratio of villus height to total mucosal thickness (V/M).

Validation of subjective assessment Twenty-five biopsies showing varying grades of abnormality were selected and independently graded by five gastroenterologists (including one of the authors) into the four grades already defined. The same biopsies were then objectively assessed by villous and crypt measurements and the two assessments compared. To judge the accuracy of distinguishing normal (grade 1) from abnormal, a control range of indices was determined by selecting the best orientated biopsies taken from just past the duodenojejunal junction from 19 patients in whom no organic disease had been found after extensive investigation. Most of these were considered to have the irritable colon syndrome. Thirty-one biopsies from these 19 patients were subjected to the objective assessment. 
Patchiness in $C D$ and $D H$ patients Details of the patients studied are shown in Table 1. All the CD patients had symptoms when first seen and all had evidence of malabsorption. In all except one of the DH patients the diagnosis was confirmed by the immunofluorescent demonstration of $\mathrm{IgA}$ in clinically uninvolved skin. Three or four biopsies were taken from just past the duodenojejunal junction in

Table 1 Details of $C D$ and $D H$ patients in whom patchiness and duodenal-jejunal variation was assessed

\begin{tabular}{lcc}
\hline Relationship to GFD & $\begin{array}{l}\text { Patients } \\
\text { (no.) }\end{array}$ & $\begin{array}{l}\text { Occasions } \\
\text { (no.) }\end{array}$ \\
\hline Coeliac disease & & \\
Before GFD only & 5 & 5 \\
After GFD only* & 17 & 25 \\
Both before and after GFD* & 9 & 20 \\
Before gluten challenge & 3 & 3 \\
After gluten challenge & & 8 \\
Dermatitis herpetiformis & 5 & 5 \\
Before GFD only & 2 & 5 \\
Both before and after GFD* & 2 & 2 \\
After GFD only* & 43 & 73 \\
Total & &
\end{tabular}

* Median interval after starting GFD-two years (range one month to 10 years)

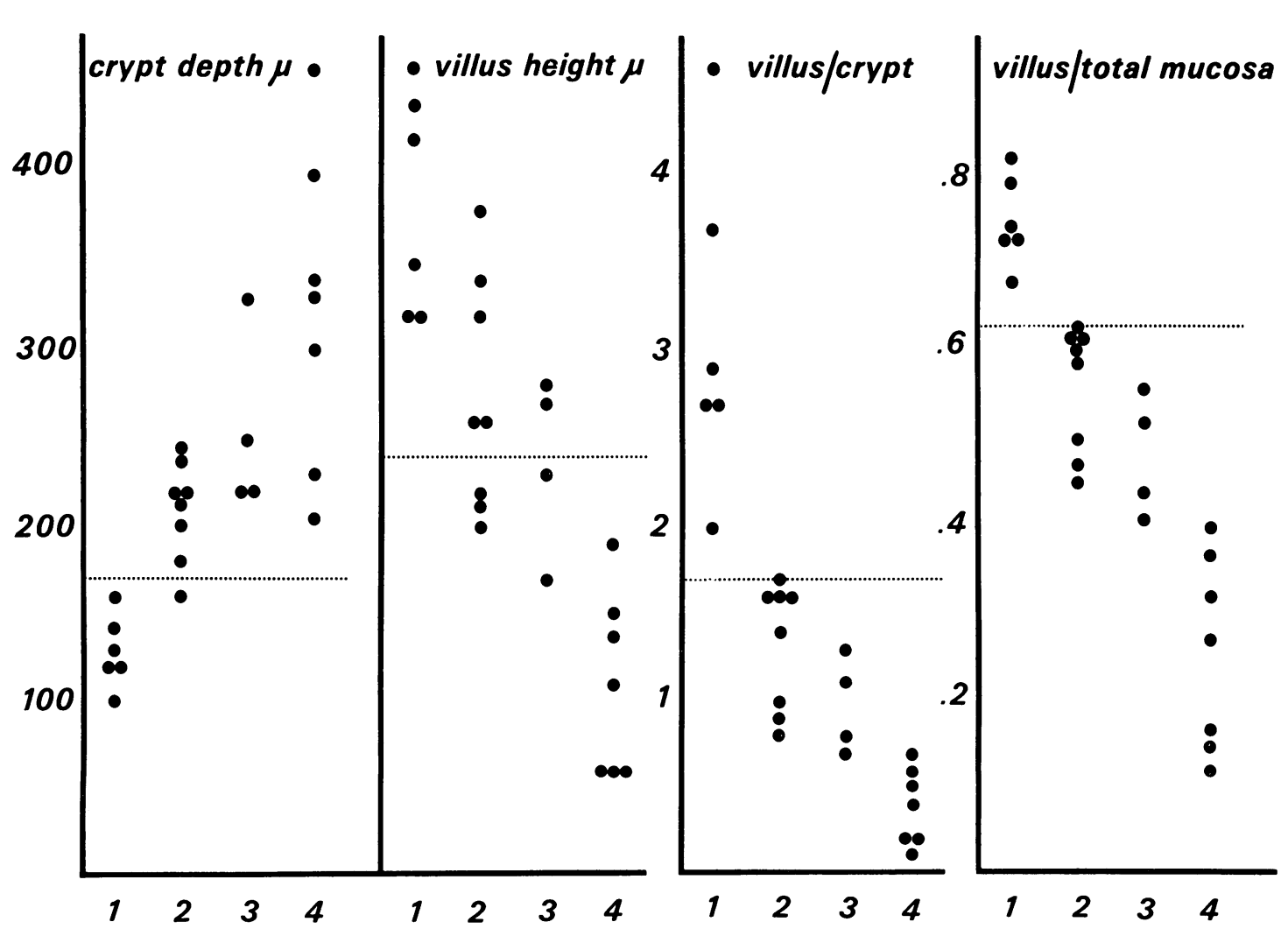

Fig. 1 Correlation of blind subjective histological assessment (grades 1, 2, 3, and 4) with objective histologica measurements. (The limits of normal are shown by the dotted lines).
Table 2 Histological grading of 25 biopsies made blind and independently by five observers

\begin{tabular}{lllll}
\hline Diagnosis & \multicolumn{3}{l}{ Histology grade } \\
\cline { 2 - 5 } & 1 & 2 & 3 & 4 \\
\hline
\end{tabular}

\begin{tabular}{|c|c|c|c|c|}
\hline \multicolumn{5}{|l|}{ Non-coeliac } \\
\hline 1 & 5 & & & \\
\hline 2 & 5 & & & \\
\hline 3 & 5 & & & \\
\hline 4 & 5 & & & \\
\hline 5 & 5 & & & \\
\hline 6 & 4 & 1 & & \\
\hline 7 Coeliac disease & 2 & 3 & & \\
\hline 8 & 2 & 3 & & \\
\hline 9 & 2 & 3 & & \\
\hline 10 & 2 & 3 & & \\
\hline 11 & & 5 & & \\
\hline 12 & & 4 & 1 & \\
\hline 13 & & 4 & 1 & \\
\hline 14 & & 4 & 1 & \\
\hline 15 & & 1 & 4 & \\
\hline 16 & & $i$ & 3 & 1 \\
\hline 17 & & & 5 & \\
\hline 18 & & & 5 & \\
\hline 19 & & & 2 & 3 \\
\hline 20 & & & 1 & 4 \\
\hline 21 & & & 1 & 4 \\
\hline 22 & & & & 5 \\
\hline 23 & & & & 5 \\
\hline 24 & & & & 5 \\
\hline 25 & & & & 5 \\
\hline
\end{tabular}


Table 3 Patchiness as assessed by stereomicroscopy and subjective histology

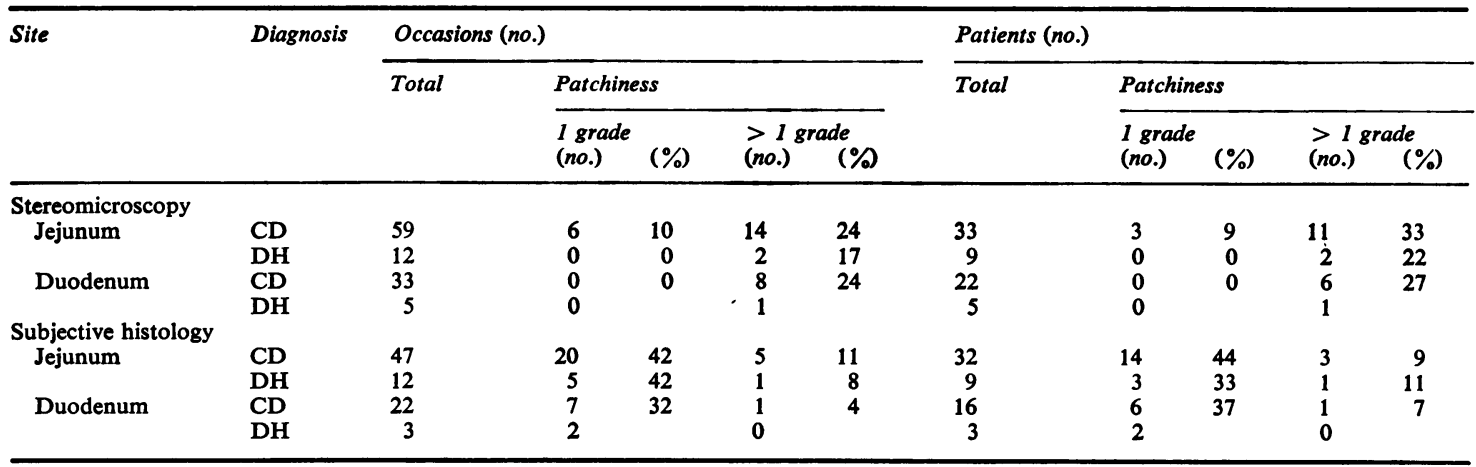

all except two patients in whom only two biopsies were taken. The number of biopsies from the second part of the duodenum was as follows: four biopsies were taken on three occasions, three on 30 occasions, and two on five occasions. On six occasions only one biopsy was taken and thus duodenal patchiness could not be assessed. Thus a total of 112 duodenal and 236 jejunal biopsies were available for assessment. All biopsies were graded by stereomicroscopy but only 225 were suitably orientated for histological assessment. Patchiness was defined as a difference of grade at one site. The frequency was separately determined for the duodenum and jejunum and for $\mathrm{CD}$ and DH patients.

\section{DUODENAL-JEJUNAL DIFFERENCES IN CONTROLS}

Sevepteen of the 19 subjects without evidence of organic disease had biopsies from both the second part of the duodenum and just past the duodenojejunal junction. All these biopsies were studied by stereo-microscopy and particular note made of (1) relative preponderance of finger and leaf-shaped villi, (2) width of leaf-shaped villi, and (3) presence of ridges.

Fourteen of the 19 subjects had satisfactory histological sections of both duodenal and jejunal biopsies, four having two suitable sections from each site. There were thus 18 matched pairs of biopsies which were objectively assessed by villous and crypt measurements and also by the presence or absence of apparent 'branching' and 'bridging' of villi in a well-orientated part of the section. The results of measurements at the two sites were compared using Student's paired $t$ test and the 'branching' and 'bridging' was compared using a $2 \times 2$ chi-squared test applying Yates' correction.

\section{DUODENAL-JEJUNAL DIFFERENCES IN CD} AND DH PATIENTS

Details of the patients studied are shown in Table 1.
The appearances of biopsies taken from just past the duodenojejunal junction and from the second part of the duodenum were compared by both stereomicroscopic and subjective histological grading. Forty-four sets of biopsies were available for comparison by stereomicroscopy and 38 by histology (six sets were unsuitable because of poor orientation). The comparison was made of the worst grade at each site.

\section{Results}

\section{VALIDATION OF SUBJECTIVE HISTOLOGICAL ASSESSMENT}

The histological grading of the 25 biopsies made by five gastroenterologists independently and without knowledge of the patients is shown in Table 2. There was complete agreement on the grading in $48 \%$ of biopsies and there was a difference of more than one grade in only one. The majority decision was accepted for each biopsy. Thus there were six grade 1 , eight grade 2 , four grade 3 , and seven grade 4 biopsies for the correlation with objective measurements of crypts and villi. The correlation was very good, as shown in Fig. 1. The means and ranges (shown in parentheses) of the measurements and indices obtained from the 31 control biopsies from 19 patients without evidence of organic disease were as follows:

Total mucosal thickness (M) $\quad 514 \mu(380-670)$ Villus height (V) $\quad . . \quad \ldots \quad 386 \mu(240-540)$ Crypt depth (C) $\quad . \quad \quad \ldots \quad 128 \mu(80-170)$

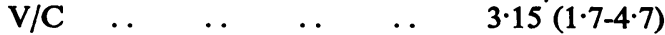
V/M $\quad . \quad \ldots \quad \ldots \quad \ldots \quad \ldots \quad 0.75(0.63-0.83)$

These results are within the range of results obtained by other workers. It can be seen in Fig. 1 that the subjective assessment gives good separation of normal from abnormal as decided by objective measurements. This separation was best with the villus to crypt and villus to mucosal thickness ratios. 


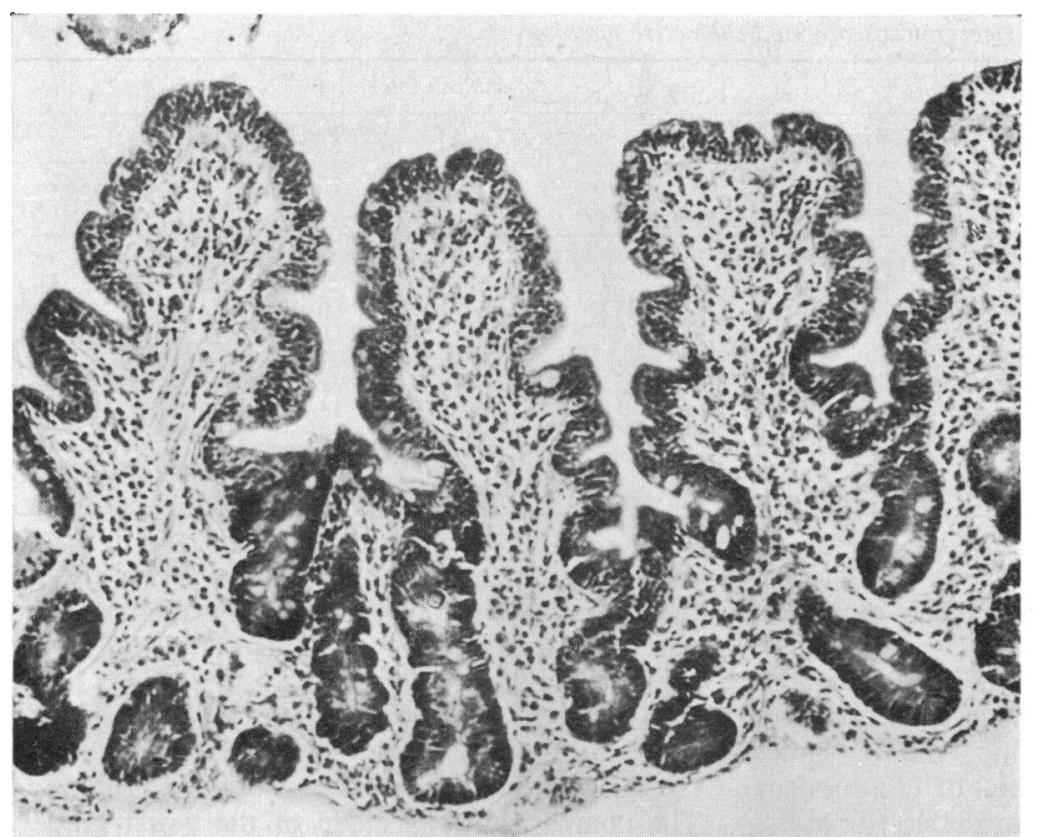

Fig. 2 Patchiness in the jejunum. Two biopsies taken at the same time from just past the duodenojejunal junction in an untreated patient with $C D$. (a) Grade 2 abnormality. (b) Grade 4 abnormality. $\times 115$.

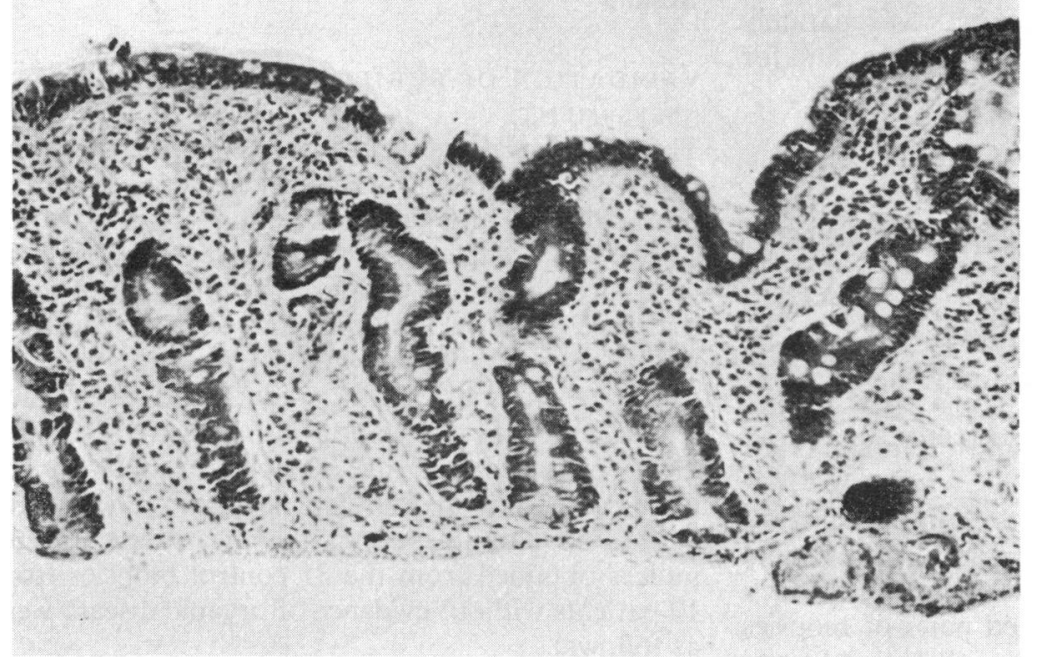

ASSESSMENT OF PATCHINESS

The incidence and degree of patchiness in the duodenum and jejunum is shown in Table 3. As assessed by stereomicroscopy patchiness occurred in $22(31 \%)$ of 71 sets of jejunal biopsies, there being a difference of one grade on six occasions, and of two grades on 14 occasions. In two patients there was a difference of three grades, from villous to flat, but the villi were not regarded as completely normal. In the duodenum, patchiness occurred in nine $(24 \%)$ of 38 sets of biopsies, there being a difference of two grades on seven occasions and of three grades on two occasions. As assessed by subjective histology, patchiness occurred in $31(52 \%)$ of 59 sets of jejunal biopsies, there being a difference of one grade on 25 occasions and of two grades on six occasions. In the duodenum patchiness occurred in $10(40 \%)$ of 25 sets of biopsies, there being a difference of 
Table 4 Comparison of objective measurements of duodenum and jejunum in 18 matched pairs from control subjects

\begin{tabular}{|c|c|c|c|c|c|}
\hline & \multicolumn{2}{|c|}{ Duodenum } & \multicolumn{2}{|c|}{ Jejunum } & \multirow{2}{*}{$\begin{array}{l}\text { Difference by } \\
\text { Student's paired } \\
\text { t test }\end{array}$} \\
\hline & Mean & $S D$ & Mean & $S D$ & \\
\hline Villous height $(\mu)$ & 312 & 62 & 398 & 72 & NS \\
\hline Crypt depth $(\mu)$ & 136 & 30 & 120 & 22 & NS \\
\hline $\mathrm{V} / \mathrm{C}$ & $2 \cdot 37$ & 0.61 & 3.45 & 0.88 & NS \\
\hline $\mathbf{V} / \mathbf{M}$ & 0.69 & 0.05 & 0.76 & 0.05 & NS \\
\hline
\end{tabular}

V/C: ratio of villous height to crypt depth.

$\mathrm{V} / \mathrm{M}$ : ratio of villous height to total mucosal thickness.

one grade in all except one in which there was a difference of two grades. Patchiness was no more frequent or severe in DH than CD. Patchiness was not confined to those on treatment with a GFD: three of 13 untreated CD patients having patchiness on stereomicroscopy and seven of 13 on histology (in one and two patients respectively the patchiness was of more than one grade); two of seven DH patients on a normal diet having patchiness on stereomicroscopy and three of seven on histology (two of the patients had patchiness of more than one grade on stereomicroscopy). Patchiness was seen at both ends of the spectrum of mucosal abnormality-for example, the variation in the jejunum as assessed by histology on 59 occasions was as follows: grade 4-3 on 10 occasions; grade 4-2 on five occasions; grade 3-2 on 11 occasions; grade 3-1 on one occasion; and grade $2-1$ on four occasions. An example of patchiness is shown in Fig. 2.

\section{DUODENAL-JEJUNAL VARIATION IN}

\section{CONTROLS}

By stereomicroscopy there was no difference of villous appearances in nine $(53 \%)$ of the 17 comparisons. In three $(18 \%)$ leaf-shaped villi were broader in the duodenum, in three $(18 \%)$ finger-shaped villi were more prominent in the jejunum. In one patient ridges were seen in the duodenum and in another ridges were seen in the jejunum. The mean and SD of the objective measurements are shown for each site in Table 4. There was no significant difference between duodenum and jejunum for any of the measurements (Student's paired $t$ test). However, the villi tended to be higher and the crypts shorter in the jejunal biopsies. There was no significant difference between the incidence of villous 'branching' and 'bridging' in well orientated parts of duodenal and jejunal biopsies. Four of the duodenal biopsies showed 'branching' compared with six of the jejunal biopsies. Three of the duodenal biopsies showed 'bridging' compared with one of the jejunal biopsies. However, if less well orientated parts of biopsies were studied, 'bridging' and 'branching' were much more common, and duodenal biopsies tended to be less well orientated, possibly because of the presence of large collections of Brunner's glands that prevented the biopsies lying flat when being mounted before fixing.

\section{DUODENAL-JEJUNAL VARIATION IN CD AND \\ DH PATIENTS}

The results of comparing the appearance of the worst duodenal biopsy with the worst jejunal biopsy on each occasion are shown in Table 5 . The differences were usually of one grade but in six a difference of more than one grade was found by stereomicroscopy and in two by subjective histology. The differences were similar in patients with $C D$ and DH. The duodenal-jejunal differences were as common in patients before treatment with a GFD as in those on treatment; the duodenal appearance being worse in three of nine CD patients and one of three DH patients, and the jejunal appearance being worse in one of the CD patients and in two of the DH patients. Figure 3 is an example of duodenaljejunal variation in which the jejunal biopsy is more severely abnormal.

\section{Discussion}

PATCHINESS

On assessment by stereomicroscopy or subjective histology (which was adequately validated) the

Table 5 Comparison of duodenal and jejunal appearances in CD and DH patients (worst appearance at each site compared)

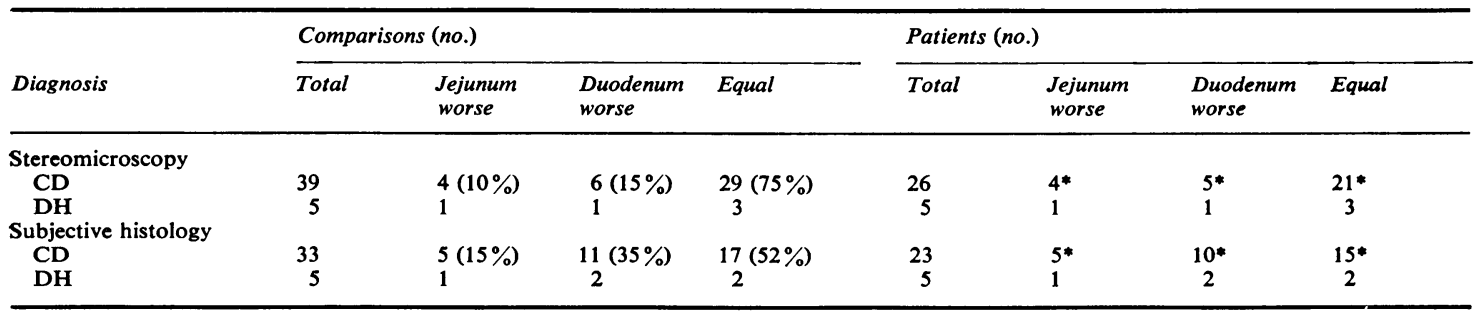

* The sum of these numbers exceeds the total number of patients as some patients exhibited a different pattern on repeat biopsy. 


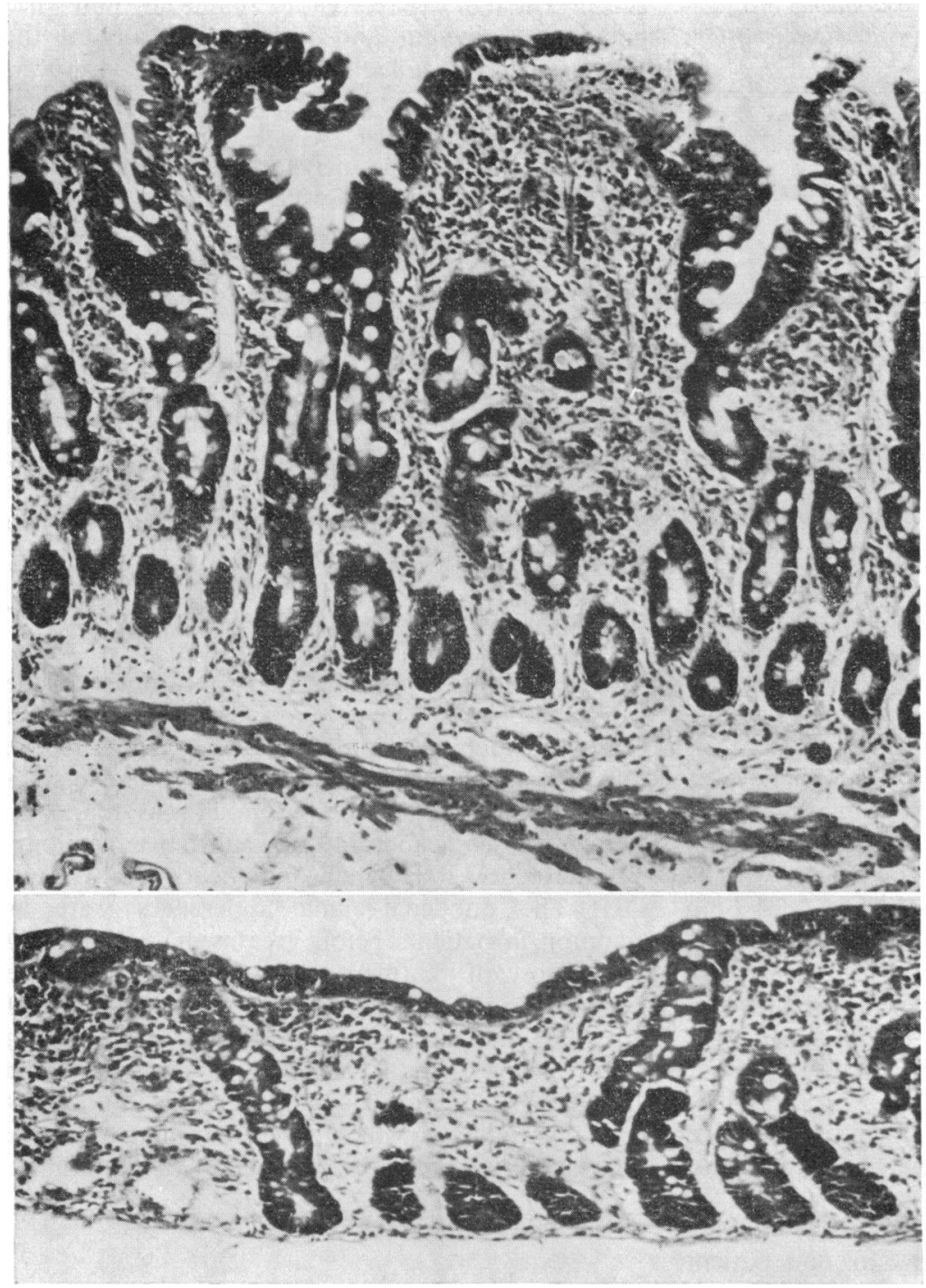

Fig. 3 Duodenal-jejunal variation. Both biopsies were taken from the same untreated $C D$ patient on the same occasion. (a) Duodenum: grade 3 abnormality. (b) Jejunum: grade 4 abnormality. $\times 125$.

frequency of patchiness was high in both duodenum and jejunum and occurred with similar frequency before and after treatment. It is possible that a difference of one grade may be due to observer error but a difference of more than that is likely to be significant. Nevertheless, it is important to know how great and how common is observer error because it would otherwise be misinterpreted. Such information was not previously available. The findings confirm the impression in the UK that patchiness is not unusual in CD. This has important implications in the assessment of a morphological response to treatment as sampling error could easily give a false impression of improvement. We therefore concur with Cooke (1968) who concluded that because of patchiness multiple biopsies are '. . . certainly necessary if any reliance is to be placed on the mucosal findings in assessing response to therapy'. Histological improvement from grade 4 to grade 1 , or stereomicroscopic improvement from flat to normal villi, at the same level, would be significant even on single biopsies as such extreme variation was not observed. However, for detecting lesser degrees of improvement, multiple biopsies are essential. It is difficult to lay down precise criteria for assessing improvement using information from 
multiple biopsies. Morphological improvement could be regarded as definite if the worst grade of multiple biopsies after treatment was better by at least one grade than the best of biopsies from the same site before treatment. The frequency of patchiness was similar in patients with $\mathrm{CD}$ and $\mathrm{DH}$, thus providing no support for the belief that this represents a difference in the mucosal lesion in the two disorders (Brow et al., 1971; Gebhard et al., 1974).

\section{DUODENAL-JEJUNAL VARIATION}

In control subjects without evidence of small bowel disease stereomicroscopy showed that the villi tended to be broader in the duodenum compared with the jejunum and this agrees with the findings of Roy-Choudhury et al. (1966). Ridges were seen in two control subjects; in one they were seen in the jejunum and in the other in the duodenum.

By objective histological assessment there was no significant difference of any of the measurements between duodenum and jejunum, although villi tended to be taller and crypts shorter in the jejunum. Rubin et al. (1960) found that objective measurements of surface epithelial density in the duodenum also did not differ significantly from those observed in the jejunum, although the duodenal villi tended to be branched, fused at the tips, or shorter and wider than jejunal villi. It was noteworthy, therefore, that sections from duodenal biopsies tended to be less well orientated (probably because of the presence of Brunner's glands which often prevent the specimens from being laid flat when mounted before fixing) and among such badly orientated sections there was a high incidence of apparent 'branching' and 'bridging' of villi which might be responsible for the impression that duodenal biopsies are more abnormal than are jejunal. However, using only wellorientated sections, there was no significant difference in the incidence of these features between duodenum and jejunum in this study.

In assessing mucosal abnormality it would therefore appear that duodenal biopsies are satisfactory as long as only well-orientated sections are used and if the rare case of massive infiltration of the mucosa with Brunner's glands is recognised (Rubin et al., 1960). Moreover, it may be that such biopsies are preferable if mild cases of $\mathrm{CD}$ are to be detected, as, in general, the mucosal abnormality of $\mathrm{CD}$ is more severe proximally. Certainly, this study has shown that, in CD patients, the duodenal appearances vary considerably from those in the jejunum-the appearances being uniform in only half, the appearance in the duodenum being worse in one-third, and, suprisingly, the jejunal appearance being worse in the remainder. The chances of detecting a mucosal abnormality are obviously greater if both sites are biopsied. It is therefore suggested that, to detect a mucosal abnormality, several biopsies should be taken from at least both these sites. In assessing response to treatment it will usually be necessary to compare multiple biopsies taken from the same level.

\section{References}

Baker, S. J., and Hughes, A. (1960). Multiple-retrieving small-intestinal biopsy tube. Lancet, 2, 686-687.

Booth, C. C. (1970). Enterocyte in coeliac disease. British Medical Journal, 3, 725-731.

Brandborg, L. L., Rubin, C. E., and Quinton, W. E. (1959). A multipurpose instrument for suction biopsy of the esophagus, stomach, small bowel, and colon. Gastroenterology, 37, 1-16.

Broitman, S. A., McCray, R. S., May, J. C., Deren, J. J., Ackroyd, F., Gottlieb, L. S., McDermott, W., and Zamcheck, N. (1970). Mastocytosis and intestinal malabsorption. American Journal of Medicine, 48, 382-389.

Brow, J. R., Parker, F., Weinstein, W. M., and Rubin, C. E. (1971). The small intestinal mucosa in dermatitis herpetiformis. Gastroenterology, 60, 355-369.

Cooke, W. T. (1968). Adult celiac disease. Progress in Gastroenterology, 1, 299-338.

Flick, A. L., Quinton, W. E., and Rubin, C E. (1961). A peroral hydraulic biopsy tube for multiple sampling at any level of the gastrointestinal tract. Gastroenterology, 40, 120-126.

Gebhard, R. L., Falchuk, Z. M., Katz, S. I., Sessoms, C., Rogentine, G. N., and Strober, W. (1974). Dermatitis herpetiformis, immunologic concomitants of small intestinal disease and relationship to histocompatibility antigen HL-A8. Journal of Clinical Investigation, 54, 98-103.

Girdwood, R. H., Williams, A. W., McManus, J. P. A., Dellipiani, A. W., Delamore, I. W., and Kershaw, P. W. (1966). Jejunal biopsy in patients with malabsorptive disease. Scottish Medical Journal, 11, 343-355.

Holmes, R., Hourihane, D. O'B., and Booth, C. C. (1961). Dissecting-microscope appearances of jejunal biopsy specimens from patients with 'idopathic steatorrhoea'. Lancet, 1, 81-83.

Hourihane, D. O'B. (1963). The histology of intestinal biopsies. Proceedings of the Royal Society of Medicine, 56, 1073-1077.

Jeffries, G. H., Steinberg, H., and Sleisenger, M. H. (1968). Chronic ulcerative (nongranulomatous) jejunitis. American Journal of Medicine, 44, 47-59.

MacDonald, W. C., Brandborg, L. L., Flick, A. L., Trier, J. S., and Rubin, C. E. (1964). Studies of celiac sprue. IV. The response of the whole length of the small bowel to a gluten-free diet. Gastroenterology, 47, 573-589.

Robinson, D. C., Watson, A. J., Wyatt, E. H., Marks, J. M., and Roberts, D. F. (1971). Incidence of small-intestinal mucosal abnormalities and of clinical coeliac disease in the relatives of children with coeliac disease. Gut, 12 , 789-793.

Roy-Choudhury, D., Cooke, W. T., Tan, D. T., Banwell, J. G., and Smits, B. J. (1966). Jejunal biopsy: criteria and significance. Scandinavian Journal of Gastroenterology, 1, $57-74$.

Rubin, C. E. (1961). Malabsorption: celiac sprue. Annual Review of Medicine, 12, 39-54.

Rubin, C. E., Brandborg, L. L., Phelps, P. C., and Taylor, H. C. (1960). Studies in celiac disease. Gastroenterology, 38, 28-49.

Rubin, C. E., and Dobbins, W. O. (1965). Peroral biopsy of the small intestine. Gastroenterology, 49, 676-697. 
Scott, G. B., Williams, M. J., and Clark, C. G. (1964). Comparison of jejunal mucosa in post-gastrectomy states, idiopathic steatorrhoea and controls using the dissecting microscope and conventional histological methods. Gut, 5, 553-562.

Spiro, H. M., Filipe, M. I., Stewart, J. S., Booth, C. C., and Pearse, A. G. E. (1964). Functional histochemistry of the small bowel mucosa in malabsorptive syndromes. Gut, 5 , 145-154.

Stevens, F. M., Lloyd, R., Egan-Mitchell, B., Mylotte, M. J., Fottrell, P. F., Wright, R., McNicholl, B., and McCarthy
C. F. (1975). Reticulin antibodies in patients with coeliac disease and their relatives. Gut, 16, 598-602.

Stewart, J. S., Pollock, D. J., Hoffbrand, A. V., Mollin, D. L., and Booth, C. C. (1967). A study of proximal and distal intestinal structure and absorptive function in idiopathic steatorrhoea. Quarterly Journal of Medicine, 36, 425-444.

Thompson, H. (1974). The small intestine at autopsy. Clinics in Gastroenterology, 3, 171-181.

Weinstein, W. M. (1974). Latent celiac sprue. Gastroenterology, 66, 489-493. 